\title{
Differences in Balance, Proprioception and Reaction Time in Land and Water Based Athletes - An Observational Study
}

\author{
Gurudut Peeyoosha, Barve Samruddhi and Kalekar Sharon
}

\begin{abstract}
Aim: The primary objective was to evaluate and compare the differences in Balance, Proprioception and Reaction time in water and land based athletes. The secondary objective was to compare between the genders. Method: 72 subjects were recruited into 2 groups as swimmers $(\mathrm{n}=36)$ and non-swimmers $(\mathrm{n}=36)$. Participants were assessed for Balance, Proprioception and Reaction time. Result: Balance and Reaction time proved to be equal in both the groups and the gender. Significant difference was noted in Swimmers and Non-swimmers for proprioception at $30^{\circ}(\mathrm{p}=0.018)$ and at $60^{\circ}(\mathrm{p}=0.006)$. Swimmer males demonstrated better proprioception at $30^{\circ}(\mathrm{p}=0.006)$ and $60^{\circ}(\mathrm{p}=0.001)$ and reaction time $(\mathrm{p}=0.003)$ than non-swimmer counterparts. Conclusion: Training medium may have a significant impact on proprioception, with aquatic medium being better than land medium. Comparison of balance, proprioception and reaction time was done where male swimmers were better than their non-swimmer counterparts. However, no such differences were found between female athletes.
\end{abstract}

\section{Gurudut Peeyoosha}

Associate Professor

Department of Orthopedic Physiotherapy

KLEU Institute of Physiotherapy, Belagavi (Karantaka) India

E-mail: peeoo.123@yahoo.com

Barve Samruddhi

BPT

Department of Orthopedic Physiotherapy

KLEU Institute of Physiotherapy, Belagavi (Karantaka) India

Kalekar Sharon

BPT

Department of Orthopedic Physiotherapy

KLEU Institute of Physiotherapy, Belagavi (Karantaka) India
Key Words: Athletes, Proprioception, Reaction Time, Balance, Physical Fitness

DOI: $10.18376 / j e s p / 2018 / v 14 / i 2 / 111304$

\section{Introduction}

Fitness is the term used to describe the ability to perform physical work by individuals (Freedson et al. 2000). For the maintenance of well-coordinated balance of fitness every individual engages themselves in some type of physical activity. Physical fitness aids in improving the health of the individuals and also helps in prevention of the diseases (Haskell et al.1985). An athlete is considered to be somebody who is proficient in any form of physical activity or sports. Fitness in athletes plays a very important role as it helps in enhancing the optimal sports performance required for the athletes (Athletes and fitness 2017). An athlete is trained for overall development of his physical fitness which is specific to his sport activities in which he competes. Physical fitness is classified into primary and secondary components. Primary including the cardiovascular fitness , body composition, flexibility etc. and secondary components comprising of balance, reaction time ,agility, proprioception , power and co-ordination. All the components of fitness are interlinked and interdependent on each other (example speed is equally dependent on the interaction of reaction time and co-ordination). Lot of factors determines the performance of the athletes. The most 


\section{Journal of Exercise Science \& Physiotherapy Vol. 14 No. 2 (July to December) 2018 \\ ISSN: 0973-2020 (Print) $\quad I_{2}$ OR Impact Factor $=6.115$ UGC Approved [no.7485] ISSN: 2454-6089 (Online)}

important factor that aids an athlete to achieve good results and to compete with participants includes training. An athlete is trained for the improvement of both primary and secondary components which inturn has an positive effect on his performance. Athletic training helps the athlete to empower and reach their potentials. Following the principle of specificity, an athlete is trained specific to his sport. Based on their specific sports, training mediums can be either water or land i.e. a swimmer is trained more specifically in water medium while a sprinter, runner are specifically trained on land medium. Differences exist in the effects of training mediums on the athletic performance. A study done by Roth et al. (2006) which compared the effect of land based and aquatic based plyometric programs which concluded that aquatic training could be an alternative approach for enhancing performance. Literature suggested that land training in athletes helps in building up competitive levels, stamina in athletes and also helps in reducing the chance soft injury but also results in high impact stress on muscles, joints thereby resulting soreness, stress fractures and injuries. Further aquatic training helps in improving strength because of the resistance of the water during training, reduced muscle soreness as compared to land training, improving flexibility in turn enhancing balance and reaction time (Wounded 2017). Another study done by Eunkuk Kim to compare aquatic based and land based exercises as early functional rehabilitation for elite athletes with acute lower extremity ligament injury concluded that aquatic exercises may provide advantages over standard land-based therapy for rapid return to athletic activities (Kim et al. 2010). Previous studies have been done to find the effects of individual components of physical fitness on the performance of athletes. However, there is dearth in literature where three major secondary components of fitness that include balance (Gerbino et al. 2007; Isles et al. 2004; Douris et al. 2003), proprioception (Hurley et al. 1998) and reaction time (Spirduso 1975: Youngen 1959) have been assessed and compared based on sports specific training media. Further, literature exists where athletes have been trained and evaluated for individual components like balance and proprioception in specific training mediums which demonstrated controversial results. Hence, it was hypothesized that significant difference may exist in the secondary components between swimmers who are trained in water medium and non-swimmer athletes who are trained on land medium. Ample literature exists were males are compared with females for balance, proprioception and reaction time. Few studies demonstrated that no difference existed between males and females while few showed males were better than females (Spirduso 1975; Hodgkins 1963). Hence, need arises where comparison has to be done between swimmer males with non- swimmer males, swimmer females with non-swimmer females. Hence, the primary objective of the study was to evaluate and find the differences inbalance, proprioception and reaction time on the training mediums between water and land athletes. The secondary objective was to compare within the genders for balance, proprioception and reaction time for both the athletic groups.

\section{Material and method}

The research design was an Observational study and study type was a Cross sectional study. The study protocol was approved by the Ethical committee (Belagavi, Karnataka) and the procedures followed were in accordance with the 1975 Declaration of Helsinki, as revised in 2013. Before participation all individuals were given written informed consent.

A formal invitation to participate in the above study was put up. A total number of 95 participants gave their informed consent after receiving the detailed information about the aims and study procedure. They were screened and 72 subjects fulfilled the inclusion criteria and were allocated into 2 groups of Swimmers and Non- Swimmers which included 36 athletes in each group. They were sub grouped according to the genders as Non-Swimmer Males (NSWM=18), Swimmer Males $(\mathrm{SWM}=18)$ and Non-Swimmer Females $(\mathrm{NSWF}=18)$, Swimmer Females $(\mathrm{SWF}=18)$. 


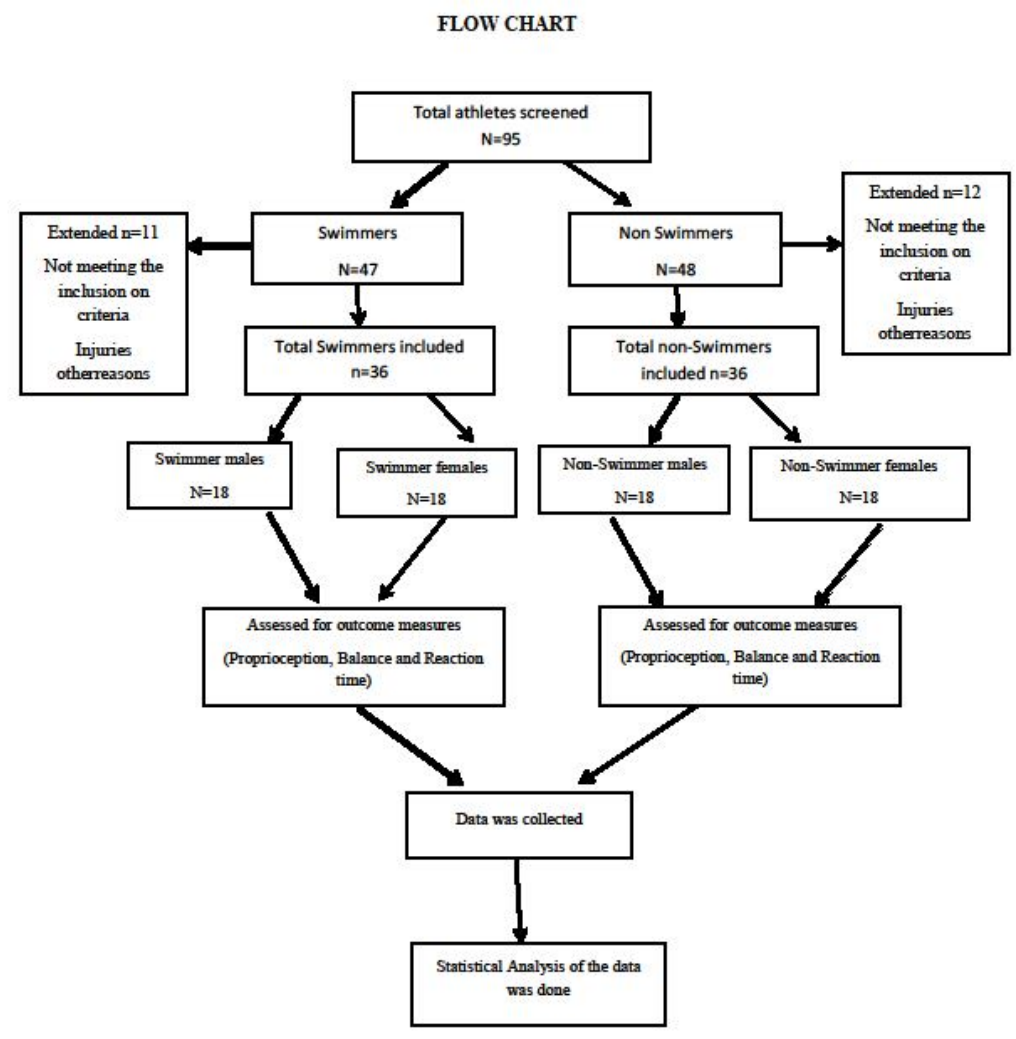

Participants were included if (i) Young adult athletes both males and females (Nulliparous) who volunteered to participate between the age group of 18 - 30 years (ii) Competitive swimmers undergoing training for at least 5days/week (iii) Competitive land athletes undergoing training for 5 days/week. The exclusion criteria were (i) History of any injury, surgery, trauma, medical illness etc. (ii) History of any vestibular conditions (iii) Physically impaired individuals (iv) Recreational athletes. All the subjects were grouped into two based on the inclusion and exclusion criteria and were screened. A brief demographic data of the subjects including the sports specialty of swimmers and non-swimmer athletes was recorded. Star Excursion Test was used to assess Balance, Digital Goniometer was used to assess proprioception and Reaction Time was assessed by Ruler Drop Test. All the 3 tests were performed for both the groups and the results were recorded. Dominant lower extremity was alone assessed by kick test.

Balance assessment was done using the star excursion balance test. In this test with the help of four strips of athletic tape of 6 to 8 feet length a plus (+) sign was drawn and a X was drawn within it so that it resembled a star shape with all the different lines separated from each other at an angle of $45^{0}$. The subjects were then explained how to perform the star excursion balance test with the 


\section{Journal of Exercise Science \& Physiotherapy Vol. 14 No. 2 (July to December) 2018 \\ ISSN: 0973-2020 (Print) $\quad I_{2}$ OR Impact Factor $=6.115$ UGC Approved [no.7485] ISSN: 2454-6089 (Online)}

dominant extremity. They were instructed to maintain a single leg stance on non- dominant leg and reach as far as possible on the dominant leg. The subjects had to reach in 8 different directions which included anterior,antero-medial, medial, postero-medial, posterior, postero-lateral, lateral and antero-lateral direction. The distance covered by the subject in all eight directions was marked and measured with measuring tape and recorded. The subjects were made to perform the test twice on the dominant lower extremity and the readings were recorded and analyzed to compare Balance of the athletes. SEBT is a reliable measure and a valid dynamic balance assessment tool. Intra-tester reliability (ICC) using this test ranged from 0.85 to 0.89 , whereas inter-tester reliability was nearly perfect, ranging from 0.97 to 1.00 (Akseki et al. 2008).

Error in proprioception assessment was done for the knee joint using a digital goniometer. It is reliable and valid instrument used to measure joint angle for accuracy $(r=0.973)$ (Ashnagar et al. 2015). The subjects were assessed in high sitting position as high sitting position is considered better than standing or supine position The subjects were given three practice sessions at target angles of $30^{\circ} 60^{\circ}$ and $90^{\circ}$ by performing passive knee flexion. After the practice sessions the subjects were asked to reproduce the given target angle on the dominant side. At each target angle, three readings were taken and the average of the three was considered for analysis (Latorre et al. 2015).

Reaction time was assessed using the Ruler Drop test. This test is simple, quick to administer, cost effective and can be easily performed in athletes. It has a good reliability $(\mathrm{r}=0.657)$ and is considered novel tool for assessment of reaction time (Yaikwawongs et al. 2009). The subjects were made to sit on the chair with his/her dominant side elbow flexed at $90^{\circ}$ with mid-pronated forearm resting on a flat horizontal table surface, with the open hand at the edge of the surface. The assessor suspended the ruler vertically with 0 centimeter marking on the ruler aligned above the web space (the space between thumb and index finger) of the subject's hand. The assessor dropped the ruler and instructed the subject to catch the falling ruler as quick as possible. The distance at which the subject caught the dropping ruler was recorded in centimeters and was converted into time using the formula, $\mathrm{t}=(2 \mathrm{~d} / \mathrm{g}) 1 / 2$. Here, $\mathrm{t}=$ reaction time; $\mathrm{d}=$ distance travelled by the ruler and $\mathrm{g}=9.81 \mathrm{~m} / \mathrm{s}^{2}$ (gravitational constant).The subjects were given three practice sessions to master the movement. After the trial sessions, the test was performed for 10 times and an average of the 10 readings was considered for analysis.

Statistical analysis for the present study was done manually as well as using statistical package of social sciences (SPSS) version 17.Structuring of statistical analysis was performed according to the purpose of the study. The intergroup comparison was done using Mann-Whitney U test. Probability values of less than 0.05 were considered statistically significant. The mean and standard deviations along with median and inter-quartile range values were reported to describe all the variables.

\section{Results}

The present study included 72 subjects who fulfilled the inclusion criteria and were allocated into 2 groups of Swimmers and Non- swimmers which included 32 athletes each and were sub grouped into males and females in each group. Swimmers versus Non-swimmers: The total mean age for non-swimmer athletes was $21.39 \pm 2.02$ and swimmer athletes was $22.83 \pm 3.75$ which was statistically seen. The data did not follow normal distribution according to the Kolmogorov Smirnoff test.

There was statistically significant difference seen on comparison of Swimmers versus Nonswimmers for proprioception error at 30 degrees ( $p$-value $=0.018)$ and at 60 degrees $(p$-value $=$ 0.006). No statistical difference was observed between the swimmers and non-swimmer athletes for proprioception error at $90^{\circ}(\mathrm{p}$-value $=0.10)$, Reaction time $(\mathrm{p}$-value $=0.065)$ and Balance $(\mathrm{p}$-value $=$ $0.28)$ (Table 1).

Gender comparison: The mean age of the athletes in Non- Swimmer males was $21.28 \pm 2.27$ and

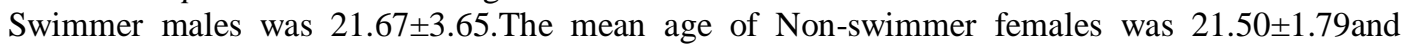


swimmer females was $24 \pm 3.58$. Statistically significant difference was seen in the gender comparison of Swimmer males versus Non swimmer males for Proprioception at $30^{\circ}$ (p-value= 0.006 ) and at $60^{\circ}$ (p-value $\left.=0.001\right)$ indicating swimmer males to have a better proprioception as compared to non-swimmer males. Reaction time demonstrated significant difference between swimmer males and non-swimmer males $(\mathrm{p}=0.003)$ indicating swimmer males being better than non-swimmer males. No statistical difference was observed for proprioception at $90^{\circ}$ with (p-value $=0.129)$ and Balance (p-value $=0.862)($ Table 2$)$. There were no significant difference seen in intergroup comparison between swimmer females and Non-swimmer females at $30^{\circ}(\mathrm{p}=0.38)$, $60^{\circ}(\mathrm{p}=0.812)$, and $90^{\circ}(\mathrm{p}=0.476)$ for Proprioception, Balance $(\mathrm{p}=0.837)$ and Reaction Time $(\mathrm{p}=0.100)$ (Table 3).

Table 1. Mean \pm SD of Proprioception, Reaction time \& Balance of Swimmers vs. Non-Swimmer Athletes

\begin{tabular}{|c|c|c|c|c|c|}
\hline Variables & Groups & Mean + SD & Median & IQR & p Value \\
\hline \multirow[t]{2}{*}{ Proprioception at $\mathbf{3 0}^{\circ}$} & SW(36) & $1.31 \pm 129$ & 0.8 & 1.8 & \multirow[t]{2}{*}{$0.018^{*}$} \\
\hline & NSW(36) & $2.68 \pm 266$ & 1.75 & 3.37 & \\
\hline \multirow[t]{2}{*}{ Proprioception at $60^{0}$} & SW(36) & $1.43 \pm 1.21$ & 0.95 & 1.13 & \multirow[t]{2}{*}{$0.006^{*}$} \\
\hline & NSW(36) & $2.84 \pm 2.68$ & 2.55 & 2.95 & \\
\hline \multirow[t]{2}{*}{ Proprioception at $90^{\circ}$} & SW(36) & $3.94 \pm 2.96$ & 3.25 & 3.15 & \multirow[t]{2}{*}{0.101} \\
\hline & NSW(36) & $6.04 \pm 5.15$ & 4.45 & 6.08 & \\
\hline \multirow[t]{2}{*}{ Reaction time(seconds) } & SW(36) & $0.18 \pm 0.07$ & 0.17 & 0.04 & \multirow[t]{2}{*}{0.065} \\
\hline & NSW(36) & $0.18 \pm 0.03$ & 0.19 & 0.04 & \\
\hline \multirow[t]{2}{*}{ Balance (centimeters) } & SW(36) & $106.95 \pm 15.4$ & 105.07 & 9.5 & \multirow[t]{2}{*}{0.28} \\
\hline & NSW(36) & $102.6 \pm 12.64$ & 100.785 & 14.85 & \\
\hline
\end{tabular}

Table 2. Mean \pm SD of Proprioception, Reaction time \& Balance of Swimmer Males vs. Non-Swimmer Males

\begin{tabular}{|c|c|c|c|c|c|}
\hline Variables & Groups & Mean \pm SD & Median & IQR & p Value \\
\hline \multirow[t]{2}{*}{ Proprioception at $3^{\circ}$} & SWM (18) & $1.22 \pm 1.14$ & 0.85 & 1.40 & \multirow{2}{*}{$0.006^{*}$} \\
\hline & NSWM (18) & $2.67 \pm 1.71$ & 2.45 & 3.18 & \\
\hline \multirow[t]{2}{*}{ Proprioception at $60^{\circ}$} & SWM (18) & $1.35 \pm 1.06$ & 1.10 & 1.03 & \multirow[b]{2}{*}{$0.001 *$} \\
\hline & NSWM (18) & $3.89 \pm 3.17$ & 3.30 & 1.55 & \\
\hline \multirow[t]{2}{*}{ Proprioception at $90^{\circ}$} & SWM (18) & $4.59 \pm 3.54$ & 3.55 & 4.90 & \multirow[b]{2}{*}{0.129} \\
\hline & NSWM (18) & $7.54 \pm 5.83$ & 6.80 & 9.90 & \\
\hline \multirow[t]{2}{*}{ Reaction time (seconds) } & SWM(18) & $0.18 \pm 0.09$ & 0.16 & 0.03 & \multirow[b]{2}{*}{$0.003^{*}$} \\
\hline & NSWM (18) & $0.19 \pm 0.01$ & 0.19 & 0.02 & \\
\hline \multirow[t]{2}{*}{ Balance (centimetres) } & SWM (18) & $110.9 \pm 20.4$ & 107.18 & 17.83 & \multirow[b]{2}{*}{0.862} \\
\hline & NSWM (18) & $106.6 \pm 10.9$ & 106.0 & 15.03 & \\
\hline
\end{tabular}

SWM: Swimmer Males ; NSWM: Non-=Swimmer Males. *significant at $\mathrm{p}<0.05$ 
Journal of Exercise Science \& Physiotherapy Vol. 14 No. 2 (July to December) 2018 ISSN: 0973-2020 (Print) $\quad I_{2}$ OR Impact Factor $=6.115$ UGC Approved [no.7485] ISSN: 2454-6089 (Online)

Table 3. Mean \pm SD of Proprioception, Reaction time \& Balance of Swimmer Females vs. Non-Swimmer Females

\begin{tabular}{|c|c|c|c|c|c|}
\hline Variables & Groups & Mean \pm SD & Median & IQR & p Value \\
\hline \multirow[t]{2}{*}{ Proprioception at $3^{\circ}$} & SWF(18) & $1.39 \pm 1.46$ & 0.66 & 2.00 & \multirow{2}{*}{0.38} \\
\hline & NSWF(18) & $2.67 \pm 3.41$ & 1.55 & 3.00 & \\
\hline \multirow[t]{2}{*}{ Proprioception at $60^{\circ}$} & SWF(18) & $1.50 \pm 1.36$ & 0.90 & 1.95 & \multirow{2}{*}{0.812} \\
\hline & NSWF(18) & $1.78 \pm 1.54$ & 1.20 & 2.25 & \\
\hline \multirow[t]{2}{*}{ Proprioception at 90 ${ }^{\circ}$} & SWF(18) & $3.28 \pm 2.11$ & 2.90 & 3.20 & \multirow{2}{*}{0.476} \\
\hline & NSWF(18) & $4.52 \pm 3.97$ & 3.95 & 2.78 & \\
\hline \multirow[t]{2}{*}{ Reaction time(seconds) } & SWF(18) & $0.17 \pm 0.02$ & 0.17 & 0.05 & \multirow{2}{*}{0.837} \\
\hline & NSWF(18) & $0.17 \pm 0.02$ & 0.17 & 0.04 & \\
\hline \multirow[t]{2}{*}{ Balance (centimetres) } & SWF(18) & $102.97 \pm 6.19$ & 102.50 & 8.56 & \multirow{2}{*}{0.100} \\
\hline & NSWF(18) & $98.54 \pm 13.15$ & 99.99 & 5.86 & \\
\hline
\end{tabular}

\section{Discussion}

SWF: Swimmer Females; NSWF: Non-Swimmer Females. *significant at $\mathrm{p}<0.05$

The present study was conducted to compare the 3 fitness components namely Proprioception, Balance and Reaction Time in Swimmer and Non- Swimmer athletes (males and females). The findings of the present study showed proprioception at 30degrees and 60degrees to be better in swimmers compared to non- swimmers. Balance and Reaction time were equal in both swimmers and non-swimmers.

Balance was assessed using the Star Excursion Balance Test and it proved to be equally good in both the swimmers and non-swimmers and within the genders with no statistical significant difference noted after analysis. In agreement to the findings of the study which was conducted on Swimmers to determine the effects of a 6-Week Dry Land Exercise Program on their performance. The results proved that the performance of the swimmers remained unchanged whether it was the aquatic environment training or on- land training (Sawdon \& Benson 2015). Another study was done to determine if aquatic and land-based balance training programs created significant improvements in levels of balance ability. The study included college aged recreational athletes and the results proved that there was no significant difference found between the groups as per their training (Spiers 2010). A study was conducted in which a comparison of static and dynamic balance was done in aquatic and land environments. Balance was assessed using star leg stance, tandem foam stance and single leg stance. The results showed that balance training can be performed equally in both land and aquatic environments. The results also proved that there was no therapeutic benefit using one environment over the other for balance training as both the environments are equally effective for the balance training (Kim et al. 2010). This equal effect was attributed to the fact that the implemented training program effectively stimulates the centrally mediated neuromuscular control mechanism which is responsible for the maintenance of balance and posture. In contrast, a study was conducted to determine the relationship between the balance ability and the athletic performance in different sports specialities. The results depicted that the gymnasts tend to have the best balance ability compared to other sports specialities like soccer players, swimmers basketball players. The study also depicted that the balance ability was related to the level of participation in the sports specific activities and the athletes with higher level of training had better balance ability. The Swimmers showed inferior balance to the soccer players since their training 


\section{Journal of Exercise Science \& Physiotherapy Vol. 14 No. 2 (July to December) 2018 ISSN: 0973-2020 (Print) $\quad I_{2}$ OR Impact Factor $=6.115$ UGC Approved [no.7485] ISSN: 2454-6089 (Online)}

does not involve the static and dynamic balance motions, and hence there is no stimulus sent to the sensory-motor systems to enhance their balance skills. Study also concluded that the training periods of all athletes may help in enhancing their strength, range of motion, and also improves the balance ability (Akseki et al. 2008). However, in the present study the balance ability of both the swimmers and non-swimmers was equally good. Both the aquatic and the land based training mediums were found to be effective in balance training. Thus, it can be concluded that athletes can undergo balance training both in water and land based mediums.

In the present study, proprioception for the knee joint was assessed at target angles of $30^{\circ}, 60^{\circ}$ and $90^{\circ}$ of knee flexion using a Digital goniometer. Proprioception was assessed in high sitting position as mentioned in accordance to previous study which states that it is a non- weight bearing position and has less errors (Hrysomallis 2011). Another study was conducted, in which proprioception at knee joint was assessed in prone and sitting position for 3 target angles. This study concluded that sitting position should be considered as compared to prone position for the assessment of the proprioception as prone position limits the error in proprioception. The present study showed that proprioception at $30^{\circ}$ and $60^{\circ}$ was better in swimmers than in non-swimmers. This difference was probably due to the difference in male swimmers and non-swimmers rather than female participants, as the statistical analysis showed significant difference in male athletes and not in female athletes. This difference could be due to the fact that effects of gravity is decreased with the aquatic mediums as the input of proprioception from the gravitational forces is neglected. In addition to this, in swimmers the motions made while swimming provides beneficial effects on improving the cardiovascular endurance, neuromuscular coordination, and stretching and elongation through lower extremities, upper limbs and trunk. Another reason could be that the movement of the motions performed in water during swimming helps to enhance the balance ability since swimmers perform this activity as a sports specific training sessions (Lokhande et al. 2013). A study was conducted for training the athletes with upper extremity injuries specifically involving the shoulder pain in aquatic based environment. This study suggests that the water is a good medium for the training of the elite athletes and for rehabilitation programmes thus water based training is a very good medium to retrain the proprioceptive senses (Lokhande et al. 2013). Various forms of exercises like aerobic exercises, stretching, resistance exercises, and functional training all can be accommodated in the aquatic based mediums for rehabilitation. The same study has given a sample rehabilitation programme in volley ball players which is a land based sports. Hence it can be concluded that sports specific training can be given in the water based mediums. Proprioception at 30degrees and 60degrees was better in Swimmer males than in non-swimmer males. A concurrent study was done on male and female athletes for proprioception. The female athletes showed proprioception deficits as compared to male athletes because females have decreased potentials as compared to males for dynamic stabilization of the knee joint with co-ordination. Knee imbalances are more common in female athletes as they demonstrate more imbalances in strength, timing of activation and decreased stiffness as compared to male athletes (Thein and Brody 2000). Another study was conducted on female intercollegiate gymnasts and healthy non- gymnasts to assess knee joint proprioception. The study showed statistically significant mean differences between the trained gymnasts, thus showing extensive training has a positive influence on knee joint proprioception as trained athletes possess enhanced neurosensory pathways which are speculated to develop as a result of long term training (Henry and Kaeding 2001). Extensive literature exists where male athletes have been compared to female athletes. To the best of authors knowledge and literature search there are no studies conducted to compare male athletes in relation to the training medium for proprioception based on sports speciality.

The significant increase in the reaction time for non-swimmer males can be attributed to the affection of proprioception in non-swimmer males. In previous literature a lot of studies are 


\section{Journal of Exercise Science \& Physiotherapy Vol. 14 No. 2 (July to December) 2018 ISSN: 0973-2020 (Print) I2OR Impact Factor =6.115 UGC Approved [no.7485] ISSN: 2454-6089 (Online)}

conducted on effects of proprioception training on balance, joint positions, muscle strength, and performance.Reaction time means the decision making process and the speed to start the movement. Thus, it is a very vital pre-requisite for athletes to give the performance. Reaction time is very important parameter, which can be influenced by various factors like age, gender, stimulus, fatigue, exercising, and training (Lephart et al. 1996). A study was done to examine effects of proprioceptive training on reaction time and hand eye coordination in volunteer students. The authors concluded that the proprioceptive training positively influenced neuromuscular processing skills improving concentration power and mind body awareness in a positive way (Ceylan and Saygin 2015). This study included assessment of reaction time for the dominant hand using sophisticated device in a noise free environment to assess for visual, audio and mixed reaction times (Ashnagar et al. 2015). But in the present study a field based on assessment method of reaction was employed. Hence, the affection of reaction time in non-swimmer males can be attributed to affection of proprioception.

\section{Conclusion}

The results demonstrated mixed results. Proprioception at 30degrees and 60degrees was found to be better in swimmers compared to non- swimmers. Further, male and female genders were compared between the groups. The results depicted that proprioception at 30degrees and 60degrees was better in swimmer males than non-swimmer males and there was no significant difference in proprioception among swimmer females and non-swimmer females. Balance was found to be equally good in the swimmer and non- swimmer males and females. Reaction time was better is the swimmer males than non-swimmer males and there was no significant difference between the swimmer females and non-swimmer females.

\section{References}

Akseki, D., Akkaya, G., Erduran, M., \& Pinar, H. (2008). Proprioception of the knee joint in patellofemoral pain syndrome. Acta Orthop Traumatol Turc, 42(5), 316-21.

Ashnagar, Z., Shadmehr, A., \&Jalaei, S. (2015). The effects of acute bout of cycling on auditory \& visual reaction times. Journal of bodywork and movement therapies, 19(2), 268-272.

Athletes and fitness - why fitness is important for athletes. Available from. www.cmhsweb.org/athletesfitness.html (Accessed on 28th July 2017)

Ceylan, H. I., \&Saygin, O. (2015). Examining the Effects of Proprioceptive Training on Coincidence Anticipation Timing, Reaction Time and Hand-Eye Coordination. Anthropologist, 20(3), 437-445.

Douris, P., Southard, V., Varga, C., Schauss, W., Gennaro, C., \& Reiss, A. (2003). The Effect of Land and Aquatic Exercise on Balance Scores in Older Adults. Journal of Geriatric Physical Therapy, 26(1), 3-6.

Freedson, P. S., Cureton, K. J., \& Heath, G. W. (2000). Status of field-based fitness testing in children and youth. Preventive medicine, 31(2), S77-S85.

Gerbino, P. G., Griffin, E. D., \&Zurakowski, D. (2007). Comparison of standing balance between female collegiate dancers and soccer players. Gait \& posture, 26(4), 501-507.

Haskell, W. L., Montoye, H. J., \& Orenstein, D. (1985). Physical activity and exercise to achieve health-related physical fitness components. Public health reports, 100(2), 202.

Henry, J. C., \&Kaeding, C. (2001). Neuromuscular differences between male and female athletes. Current women's health reports, 1(3), 241-244.

Hrysomallis, C. (2011). Balance ability and athletic performance. Sports medicine, 41(3), 221-232.

Hurley, M. V., Rees, J., \&Newham, D. J. (1998). Quadriceps function, proprioceptive acuity and functional performance in healthy young, middle-aged and elderly subjects. Age and ageing, 27(1), 55-62.

Isles, R. C., Choy, N. L., Steer, M., \&Nitz, J. C. (2004). Normal values of balance tests in women aged 2080. Journal of the American Geriatrics Society, 52(8), 1367-1372.

Kim, E., Kim, T., Kang, H., Lee, J., \& Childers, M. K. (2010). Aquatic versus land-based exercises as early functional rehabilitation for elite athletes with acute lower extremity ligament injury: a pilot study. $P M \& R, 2(8), 703-712$. 
Journal of Exercise Science \& Physiotherapy Vol. 14 No. 2 (July to December) 2018

ISSN: 0973-2020 (Print) $\mathrm{I}_{2}$ OR Impact Factor $=6.115$ UGC Approved [no.7485] ISSN: 2454-6089 (Online)

LatorreRomán, P. Á., Mora López, D., Fernández Sánchez, M., Salas Sánchez, J., Moriana Coronas, F., \&García-Pinillos, F. (2015). Test-retest reliability of a field-based physical fitness assessment for children aged 3-6 years. Nutricionhospitalaria, 32(4).

Lephart, S. M., Giraldo, J. L., Borsa, P. A., \& Fu, F. H. (1996). Knee joint proprioception: a comparison between female intercollegiate gymnasts and controls. Knee surgery, sports traumatology, arthroscopy, 4(2), 121-124.

Lokhande, M. V., Shetye, J., Mehta, A., \&Deo, M. V. (2013). Assessment of knee joint proprioception in weight bearing and in non-weight bearing positions in normal subjects. JKIMSU, 2(2), 94-101.

Roth, A. E., Miller, M. G., Ricard, M., Ritenour, D., \& Chapman, B. L. (2006). Comparisons of static and dynamic balance following training in aquatic and land environments. Journal of Sport Rehabilitation, 15(4), 299-311.

Sawdon-Bea, J., \& Benson, J. (2015). The effects of a 6-week dry land exercise program for high school swimmers. Journal of Physical Education and Sports Management, 2, 1-17.

Spiers, S. (2010). Comparison of the effects of aquatic and land-based balance training programs on the proprioception of college-aged recreational athletes (Doctoral dissertation).

Spirduso, W. W. (1975). Reaction and movement time as a function of age and physical activity level. Journal of Gerontology, 30(4), 435-440.

Thein, J. M., \& Brody, L. T. (2000). Aquatic-based rehabilitation and training for the shoulder. Journal of athletic training, 35(3), 382.

Wounded Warrior Project,Inc.[Internet] Wounded Warriors http://www.woundedwarriorproject.org/programs/physical-health-wellness/fitness/the-benefits-ofaquatic-exercise-and-deep-water-running.aspx (accessed on 3 January 2017).

Yaikwawongs, N., Limpaphayom, N., \&Wilairatana, V. (2009). Reliability of digital compass goniometer in knee joint range of motion measurement. Medical journal of the Medical Association of Thailand, 92(4), 517.

Youngen, L. (1959). A comparison of reaction and movement times of women athletes and nonathletes. Research Quarterly. American Association for Health, Physical Education and Recreation, 30(3), 349-355.

Conflict of Interest: None declared 